\title{
Achieving net-zero emissions through the reframing of UK national targets in the post-Paris Agreement era
}

Steve Pye, Francis G. N. Li, James Price and Birgit Fais

Nature Energy 2, 17024 (2017); published online 6 March 2017; corrected after print 15 May 2017.

In the version of this Article originally published, the second affiliation for Steve Pye was omitted. The affiliation has now been added. 